\title{
Molecular Weights of Thermally Degraded Polymethyl Methacrylate
}

\author{
Victor E. Hart
}

\begin{abstract}
Molecular weights of polymer residues from the thermal degradation in vacuum of two polymethyl methacrylate samples, one polymerized with benzoyl peroxide and the other without any initiator, have been obtained over wide extents of degradation at various constant temperatures. A linear relation, dependent upon the temperature but independent of rate of volatilization and rate of change of $1 / M_{w}$, is found when $\log R$ is plotted against $1 / M_{w} . \quad\left(R=\right.$ ratio of residue weight to original specimen weight, and $M_{w}=$ weight-average molecular weight.) The results are explained on the basis of a free-radical chain-reaction mechanism in which intermolecular chain transfer (activation energy $=29$ kilocalories) is the primary, or perhaps the sole, cause of the molecular-weight decrease.
\end{abstract}

\section{Introduction}

Polymethyl methacrylate is of particular interest in the study of polymer degradation because the products volatilized as a result of the thermal decomposition in vacuum consist almost entirely of the monomer, and also because the molecular weights of the residues can be conveniently measured.

Relatively little has been reported in the literature $[1 \text { to } 6]^{1}$ with regard to the degradation of polymethyl methacrylate prepared without the use of any initiator. Madorsky [1] and Straus and Madorsky [2] found such a polymethyl methacrylate to be considerably more stable (in terms of volatilization rates) at elevated temperatures in vacuum than a sample polymerized with the aid of benzoyl peroxide. They also found that in the course of pyrolysis, the residue of the benzoyl peroxidepolymerized polymer eventually achieved a stability (after about $50 \%$ of volatilization) comparable to that of the polymer polymerized without any initiator.

The main object of the present work was to determine molecular weights of the above two polymethyl methacrylate samples as functions of extent of volatilization at constant temperature for thermal degradation in vacuum. Also, it was desired to elucidate the nature of the temperature effect. In the case of the benzoyl peroxide-polymerized sample, it was of particular interest to obtain data beyond the first 50 percent of volatilization, where complicating effects of benzoyl peroxide might no longer be present.

\section{Polymethyl Methacrylate Samples}

Sample A was analyzed and found to contain approximately 0.6 percent of benzoyl peroxide. Its weight-average molecular weight was $146,000{ }^{2}$ The monomer used in the preparation of sample B was degassed and sealed in an evacuated tube without any initiator. It was allowed to polymerize in bulk at $-25^{\circ}$ to $-35^{\circ} \mathrm{C}$. The weight-average molecular weight of the resulting polymer was 5,030,000 (see footnote 2).

\footnotetext{
1 Figures in brackets indicate the literature references at the end of this paper. 2 The molecular weights were determined by Louis Williams of the Bureau, using the light-scattering method.
}

\section{Experimental Methods}

The vacuum apparatus used in pyrolysis was that described previously [7] for the study of the rates of volatilization of polytetrafluoroethylene by the gaspressure method. The specimen was inserted into a weighed Pyrex glass tube, $45 \mathrm{~mm}$ long and $6 \mathrm{~mm}$ inside diameter with one end closed, and was weighed on a semimicrobalance. The pyrolysis apparatus, with specimen in place, was kept under vacuum overnight to minimize desorption of gases from the walls of the apparatus during pyrolysis. The cylindrical external heater was so constructed that an essentially uniform temperature could be maintained over the entire specimen. The heater, after being kept at the desired temperature overnight, was moved into position in two steps, 10 min apart, during which time the current was increased by a predetermined amount and duration. The specimen reached the desired temperature in about 2 min after the heater was placed in final position. The temperature was kept within $\pm 0.5^{\circ} \mathrm{C}$. The volatile products were condensed during pyrolysis in a liquid-nitrogen trap located between the specimen and the mercuryvapor pump.

The polymer residue was dissolved out of the Pyrex glass tube with a minimum amount of distilled ACS-grade chloroform by using the small reflux apparatus shown in figure 1. Moderate reflux rates were used to avoid foaming, and complete solution usually occurred in less than 2 hrs. The Pyrex tube was then weighed to make certain that the residue was completely dissolved. The solution was allowed to stand overnight prior to viscosity measurement.

One milliliter of solution was required for an intrinsic viscosity determination. The semimicrodilution viscometer and the techniques used have been described by the author in a previous publication [8]. Kinetic-energy corrections and errors due to drainage, surface tension, solvent evaporation, and the effects of shear rate of the polymethyl methacrylate solutions were negligible with this viscometer. The temperature of the water bath was maintained at $27.30 \pm 0.01^{\circ} \mathrm{C}$. The flow time of the solvent was $195.7 \mathrm{sec}$. The latter was redetermined after each intrinsic-viscosity measurement, and_at 


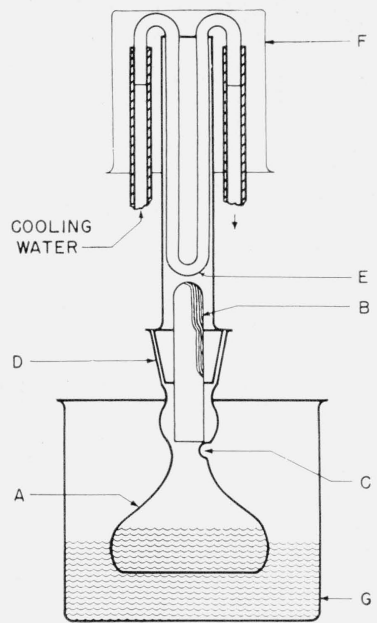

Figure 1. Dissolving fused polymer from a tube.

A, Weighing bottle containing solvent; $B$, tube containing fused polymer; $\mathrm{C}$, indentation in the glass; $\mathrm{D}$, tapered groundglass connection; $\mathrm{E}$, condenser tube; $\mathrm{F}$, beaker for keeping out dust; $G$, heating bath.

no time was it found to have changed. Average deviation of flow time for solution reruns was less than 0.1 percent, and at least one rerun was always made.

A graphic method described previously by the author [9] was used for accurately calculating intrinsic viscosity from a single specific-viscosity determination. This method is applicable to a wide range of specific viscosities. However, for convenience in measuring the specific viscosity, a value close to 1.0 was generally obtained by adjusting the size of the original polymer specimen and the concentration of the solution according to predictions based upon the trends of the data.

\section{Results}

The weight-average molecular weight, $M_{w}$, was calculated from the intrinsic viscosity, $[\eta]$, using the equation $[\eta]=3.54 \times 10^{-5} M_{w}{ }^{0.843}$. The constants in this equation were calculated from intrinsic-viscosity and light-scattering molecular-weight measurements of the original polymethyl methacrylate samples A and B. The above equation agrees satisfactorily with those reported in the literature for polymethyl methacrylate in chloroform [10,11].

In table 1 are listed molecular weights of residues from pyrolysis of the two polymethyl methacrylate samples. Prior to pyrolysis at $320^{\circ}$, each specimen of sample A was preheated by raising it in a consistent manner from room temperature to $260^{\circ}$ in $55 \mathrm{~min}$ in the vacuum apparatus. This prevented excessively rapid initial volatilization at $320^{\circ} \mathrm{C}$.

In figure 2, weight-average molecular weight is plotted in terms of percentage of the original molecular weight against percentage volatilized at $320^{\circ}$ $\mathrm{C}$ and at $150^{\circ} \mathrm{C}$. Because of a significant temperature effect, it would be incorrect to join the two curves for sample A.

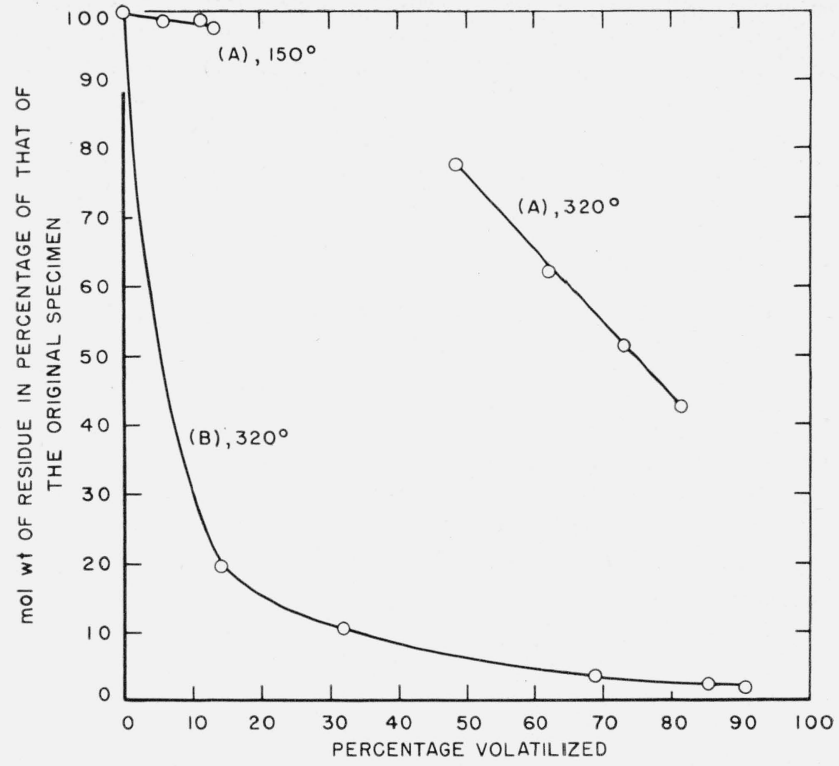

Figure 2. Effect of degradation on the molecular weight of the residue.

The nature of the molecular-weight change is better illustrated in figure 3 , where $\log R$ is plotted against $1 / M_{w}, R$ being the ratio of the residue weight to the weight of the original specimen. Straight lines result, the slopes of which depend upon the temperature. (The first $48 \%$ of the volatilization of sample $\mathrm{A}$ in the experiments at $320^{\circ} \mathrm{C}$ occurred at a high rate, before the temperature of $320^{\circ} \mathrm{C}$ could

TABLE 1. Molecular weights of residues from pyrolysis of polymethyl meihacrylate

\begin{tabular}{|c|c|c|c|c|c|}
\hline \multicolumn{4}{|c|}{ Pyrolysis } & \multicolumn{2}{|c|}{ Residue } \\
\hline $\begin{array}{l}\text { Tempera- } \\
\text { ture }\end{array}$ & $\begin{array}{l}\text { Specimen } \\
\text { weight }\end{array}$ & Duration & $\begin{array}{l}\text { Volatiliza- } \\
\text { tion }\end{array}$ & {$[\eta]$} & $M_{w}$ \\
\hline \multicolumn{6}{|c|}{ Sample A } \\
\hline${ }^{c} C$ & $m g$ & $\min$ & $\%$ & 0.801 & 146,000 \\
\hline 150 & 82.00 & 40 & 5.89 & $.7 \mathrm{Q} 2$ & 144,400 \\
\hline 150 & 78.32 & 120 & 11.13 & $.7 \div 3$ & 144,800 \\
\hline 150 & 69.22 & 300 & 13. 25 & .785 & 143,000 \\
\hline 320 & 99.96 & 10 & 48. 57 & .646 & 113,500 \\
\hline 320 & 196.90 & 50 & 62.07 & .538 & 91,300 \\
\hline 320 & 201. 14 & 100 & 73. 16 & .459 & 75,600 \\
\hline 320 & 204.56 & 180 & 81.43 & .391 & 62,500 \\
\hline \multicolumn{6}{|c|}{ Sample B } \\
\hline 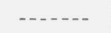 & 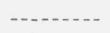 & . & 0 & 15.8 & $5,030,000$ \\
\hline 300 & 48. 75 & 450 & 52. 70 & 1. 854 & 398,000 \\
\hline 300 & 147.00 & 810 & 68.53 & 1. 302 & 260,600 \\
\hline 320 & 10.75 & 10 & 14.14 & 4. 92 & $1,000,000$ \\
\hline 320 & 28. 77 & 30 & 31.87 & 2. 41 & 541,000 \\
\hline 320 & 99.91 & 136 & 68.85 & 0.962 & 182,000 \\
\hline 320 & 136.90 & 300 & 85.30 & .680 & 120,600 \\
\hline 320 & 240.80 & 420 & 90.69 & .549 & 93,600 \\
\hline 340 & 79.68 & 10 & 41.88 & 1. 734 & 366,100 \\
\hline 340 & 132. 27 & 40 & 82.78 & 0.670 & 118,400 \\
\hline 340 & 101.77 & 48 & 84.74 & .608 & 105,600 \\
\hline
\end{tabular}


be reached. For this reason it was experimentally impracticable to determine the molecular weights within this range of volatilization at this temperature.) From figure 3 it may be inferred that in the type of plot shown in figure 2, the higher the molecular weight, the more abruptly the molecularweight curve drops, and also the higher the temperature, the more rapid the drop.

\section{Theory for $\log R$ as a Function of $1 / M_{w}$}

Defining: $R=m / m_{o}$, where $m=$ weight of specimen remaining at time, $t$, and $m_{0}=$ original weight of specimen; $\quad K_{2}=(-100 / R)(d R / d t)=-230.3 \quad(d \quad l o g$. $R) / d t=$ rate of volatilization in percentage of the residue per minute; $K_{3}=d\left(1 / M_{w}\right) / d t=$ rate of change of reciprocal weight-average molecular weight. As a consequence of the definitions,

$$
\frac{K_{2}}{K_{3}}=-230.3 \frac{d \log R}{d\left(1 / M_{w}\right)} .
$$

The object of the ensuing treatment is to indicate why $K_{2} / K_{3}$ might be expected to be very nearly constant for a given pyrolysis temperature.

A free-radical chain-reaction mechanism (stationary type) is assumed, in which the entire drop in molecular weight during pyrolysis is due to an intermolecular chain-transfer process. The initiation step of the chain reaction may be ignored, as no kinetic expression for this step is required for the present purpose.

The volatilization products of the thermal decomposition of polymethyl methacrylate consist almost entirely of the monomer [2]. As is customary in this case, it is assumed that each polymeric free radical resulting from the initiation process decreases in size by the consecutive splitting off of monomer units from its free-radical end (depropagation). Symbolically, for kinetic analysis

$$
\begin{aligned}
& P_{\dot{b}} \stackrel{k_{d}}{\rightarrow} P_{\dot{b}-1}+M \uparrow \\
& P_{\dot{b}-1} \stackrel{k_{d}}{\rightarrow} P_{\dot{b}-2}+M \uparrow \\
& \text { etc., }
\end{aligned}
$$

where $P_{\dot{b}}$ is a polymeric free radical of degree of polymerization, $b$, and $M$ represents a monomer molecule.

If $K_{d}$ is the number of monomer units splitting off per gram of residue per minute,

$$
K_{d}=k_{d}[P \cdot \mathbf{i}],
$$

where $[P \cdot]$ is the number of free radicals per gram of residue, and $\mathrm{k}_{d}$ is the depropagation-rate constant. Since this depropagation step contributes almost the entire amount of the volatilization products, the rate of volatilization, $K_{2}$, may be expressed similarly:

$$
K_{2}=k_{d}[P \cdot] \frac{100.1 \times 100}{N},
$$

where $N$ is the Avogadro number. (The molecular weight of the monomer is 100.1 .)

The chain-transfer mechanism is assumed to be of the usual hydrogen-transfer type, in which a freeradical end acquires a hydrogen atom from a carbon atom in the backbone of another chain and becomes a polymer molecule with a saturated end, $S$. The molecule attacked splits into two fragments, one of which forms an unsaturated end, $U$, and the other fragment contains a free-radical end. The latter polymer fragment commences to split off monomer. The transfer process may be represented for kinetic analysis as follows (intermolecular type):

$$
\begin{aligned}
& P_{\dot{b}-z}+P_{x} \mathrm{H} P_{y} \stackrel{k_{t \tau}}{\longrightarrow} P_{b-z} S+P_{\dot{x}} P_{y} \\
& P_{\dot{x}} P_{y} \rightarrow P_{\dot{x}}+P_{y} U \text { (rapid step), }
\end{aligned}
$$

where $\mathrm{H}$ is the transfer hydrogen, $z$ is the number of monomer units split off from the free radical prior to the transfer act, and $P_{x}$ and $P_{y}$ represent the two polymeric fragments formed from the molecule attacked at random, $P_{x} \mathrm{H} P_{y}$.

If $K_{t r}$ is the number of transfer acts occurring per gram of residue per minute, $k_{t r}$ is the transfer-rate constant, and $[\mathrm{H}]$ is the number of points at which transfer can occur per gram of residue, which is constant, then

$$
K_{t r}=k_{t r}[P \cdot][\mathrm{H}] .
$$

Because intermolecular chain transfer is assumed to be the sole cause of the molecular-weight decrease, it follows, as a close approximation within the experimental ranges of volatilization at $300^{\circ}$ to $340^{\circ}$ C, that

$$
\frac{d\left(\frac{N}{M_{n}}\right)}{d t}=K_{t r}
$$

where $M_{n}$ is the number-average molecular weight. The following is also assumed as a good approximation within these experimental ranges [12]:

$$
\frac{M_{n}}{M_{w}}=g=\text { constant. }
$$

Substituting for $M_{n}$ and $K_{t r}$ in eq (5),

$$
K_{3}=\frac{g}{N} \times k_{t r}[P \cdot][\mathrm{H}] .
$$

Termination of a reaction chain is assumed to occur merely when a free radical becomes reduced by depropagation to monomer size or to the point where it escapes as a polymeric fragment along with the monomer being evolved. This may be called "termination by free-radical depletion." 


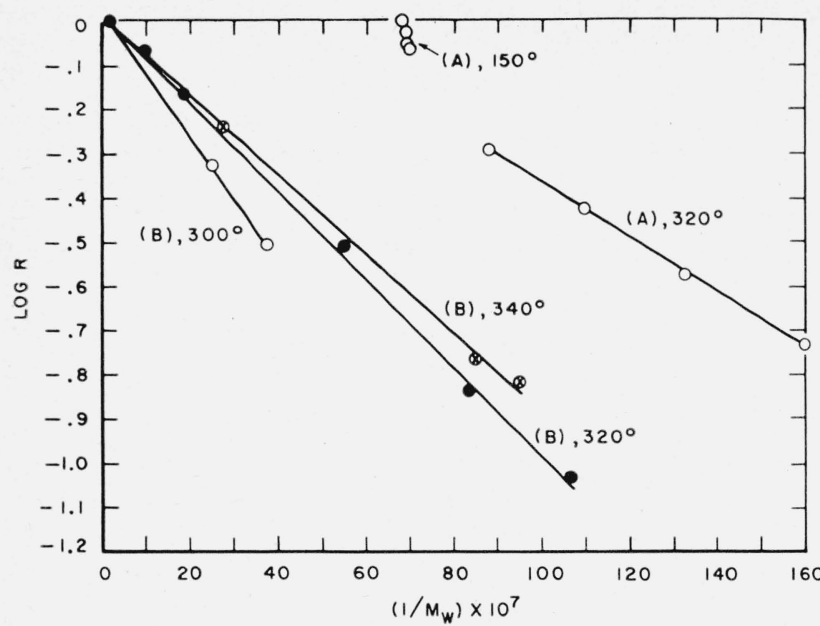

Figure 3. $\log R$ versus $1 / M_{w}$.

$\mathrm{R}$ is the weight of the residue at any given pyrolysis time divided by the original specimen weight,

Eliminating $[P \cdot]$ between eq (3) and (7),

$$
\frac{K_{2}}{K_{3}}=\frac{k_{d} \times 1.001 \times 10^{4}}{g k_{l r}[\mathrm{H}]}
$$

It has been assumed that $g$ and $[\mathrm{H}]$ are constant. If it is further assumed that the rate constants, $k_{d}$ and $k_{t r}$, are indeed constant at a fixed temperature, it follows that $K_{2} / K_{3}$ should be constant, and referring to eq (1), that $(d \log R) /\left[d\left(1 / M_{w}\right)\right]$ should be constant. It is clear that the theory predicts for a given sample that a plot of $\log \dot{R}$ against $1 / M_{w}$ (as in fig. 3) will be a straight line for pyrolysis at a fixed temperature, independent of change in magnitude of $K_{2}$ and $K_{3}$, and that the slope of the plot will vary with the temperature, depending on the ratio, $k_{a} / k_{t r}$.

For the purpose of testing the freedom of the experimental slopes from dependence upon the magnitude of the rates, $K_{2}$ and $K_{3}$, it is fortunate that these rates have been found to vary with extent of degradation at $320^{\circ} \mathrm{C}$. Both samples $\mathrm{A}$ and $\mathrm{B}$ showed decreasing rates, and at the end of the experimental ranges of pyrolysis the rates were about a third of the initial values in both cases. These results further support intermolecular chain transfer as the primary, or perhaps the sole, cause of the molecular-weight change. The experimental rate curves covering wide extents of degradation at $320^{\circ} \mathrm{C}$, together with studies of the effects of benzoyl peroxide and of inhibitors, are planned for inclusion in a separate publication, in which the initiation mechanism may be elucidated.

In the case of sample A, 48 percent of volatilization occurs before the residue can reach $320^{\circ}$. If the actual values of $\log R$ versus $1 / M_{w}$ for those specimens of $\mathrm{A}$ for which data were obtained at $320^{\circ}$ were plotted for the first 48 percent of volatilization, the curve would reflect the lower temperatures in this range and the corresponding changes in slope. Therefore, the straight line obtained at $320^{\circ}$ does not extrapolate back to the initial molecular weight at $\log R=0$. Sample B incurs about 8 percent of volatilization before it can reach $320^{\circ}$. However, this amount is quite small for initial curvature to be indicated, and the straight lines do appear in this case to extrapolate to the initial molecular weight at $\log R=0$.

The slope for sample B is 1.67 times that for sample $\mathrm{A}$ at $320^{\circ} \mathrm{C}$. No satisfactory explanation is available at present for this difference. However, some heterogeneity in the molecular structure is suggested in the case of sample $A$, at least after the temperature of the sample has been raised sufficiently high. A possible reaction of some sort involving benzoyl peroxide and groups along the polymer chain, and resulting in a few points at which the probability of transfer is greatly enhanced, may be considered.

\section{Activation Energies}

Rearranging eq (8),

$$
k_{t r}=\frac{1.001 \times 10^{4}}{g[\mathrm{H}]} \times \frac{K_{3} k_{d}}{K_{2}} .
$$

The constants may be combined and replaced with $C_{1}$ :

$$
k_{t r}=C_{1} \times \frac{K_{3} k_{d}}{K_{2}} .
$$

Taking the logarithm of the latter expression and then the derivative with respect to $1 / T$, where $T$ is the absolute temperature,

$$
\frac{d \log k_{t r}}{d(1 / T)}=\frac{d \log K_{3}}{d(1 / T)}-\frac{d \log K_{2}}{d(1 / T)}+\frac{d \log k_{d}}{d(1 / T)} .
$$

It follows that

$$
E_{t r}=E_{3}-E_{2}+E_{d}
$$

where $E_{t r}$ and $E_{d}$ are the activation energies for the chain transfer act and the depropagation act, respectively, and $E_{3}$ and $E_{2}$ are the apparent activation energies corresponding to the initial $K_{3}$ and $K_{2}$ rates.

If $s$ represents the slope, $(d \log R) /\left[d\left(1 / M_{w}\right)\right]$, from eq (1)

$$
\frac{K_{3}}{K_{2}}=\frac{1}{-s 230.3}
$$

Taking the common logarithm of this expression, and then the derivative with respect to the reciprocal of the absolute temperature,

$$
\frac{d \log K_{3}}{d(1 / T)}-\frac{d \log K_{2}}{d(1 / T)}=-\frac{d \log (-s)}{d(1 / T)} .
$$

If each term is multiplied by $-4.576 \times 10^{-3}$ (the molar gas constant appearing in the Arrhenius equa- 
tion, $1.9872 \times 10^{-3} \mathrm{kcal}^{\mathrm{mole}} \mathrm{e}^{-1}$ degree $^{-1}$, multiplied by -2.3026 ), eq. (13) becomes, for initial rates].

$$
E_{3}-E_{2}=\left(4.576 \times 10^{-3}\right) \frac{d \log (-s)}{d(1 / T)},
$$

where $E_{3}$ and $E_{2}$ are expressed in terms of kilocalories per mole.

From eq (11) one would expect $E_{3}-E_{2}$ to be constant within the experimental temperature range. Equation (14) could then be written as follows, for evaluation between temperatures, $T_{1}$ and $T_{2}$ :

$$
E_{3}-E_{2}=\left(4.576 \times 10^{-3}\right) \frac{\log \frac{s_{2}}{s_{1}}}{\frac{1}{T_{2}}-\frac{1}{T_{1}}} .
$$

The apparent activation energies, $E_{3}$ and $E_{2}$, are based upon initial rates, and $s$ should therefore be the initial slope. As $s$ remains constant at constant temperature, however, the accuracy of the value of $E_{3}-E_{2}$ calculated through the use of eq (15) should in the present case be greater than that which might be obtained by the usual method of determining each activation energy separately from the initial rates.

The following experimental values of $s$ are found for sample B: $-13.79 \times 10^{4}$ at $300^{\circ} \mathrm{C} ;-10.07 \times 10^{4}$ at $320^{\circ} \mathrm{C}$; and $-9.04 \times 10^{4}$ at $340^{\circ} \mathrm{C}$. The resulting values calculated for $E_{3}-E_{2}$ are $10.6 \mathrm{kcal}$ at $300^{\circ}$ to $320^{\circ} \mathrm{C}$ and $3.9 \mathrm{kcal}$ at $320^{\circ}$ to $340^{\circ} \mathrm{C}$. There seems to be no entirely satisfactory explanation for this difference, but the figure calculated for the $300^{\circ}$ to $320^{\circ} \mathrm{C}$ range may be preferred because the rates of volatilization at $340^{\circ} \mathrm{C}$ are quite high from the experimental standpoint. It cannot be ascertained from the present data whether a systematic experimental error is actually involved at $340^{\circ} \mathrm{C}$ or whether a change in mechanism is indicated.

Cowley and Melville estimated the activation energy for depropagation, $E_{d}$, to be $18.5 \mathrm{kcal} / \mathrm{mole}$ from figures for the heat of polymerization $(-13.0 \mathrm{kcal})$, and for the activation energy of propagation (5.5 kcal) [3]. If this value for $E_{\vec{a}}$ and the value for $E_{3}-E_{2}$ in the range $300^{\circ}$ to $320^{\circ} \mathrm{C}$ are substituted in eq (11), a value of $29 \mathrm{kcal} / \mathrm{mole}$ is obtained as an estimate of the activation energy for the transfer act in the degradation of sample $\mathrm{B}$.

\section{References}

[1] S. L. Madorsky, J. Polymer Sci. 11, 491 (1953).

[2] S. Straus and S. L. Madorsky, J. Research NBS 50, 165 . (1953) RP2405.

[3] P. R. E. J. Cowley and H. W. Melville, Proc. Roy. Soc. (London) [A] 210, 461 (1951).

[4] P. R. E. J. Cowley and H. W. Melville, Proc. Rov. Soc (London) [A] 211, 320 (1952).

[5] N. Grassie and H. W. Melville, Proc. Roy. Soc. (London). [A] 199, 1 (1949).

[6] S. Bywater, J. Phys. Chem. 57, 879 (1953).

[7] S. L. Madorsky, V. E. Hart, S. Straus, and V. A. Sedlak, J. Research NBS 51, 327 (1953) RP2461.

[8] V. E. Hart, J. Polymer Sci. 17, 207 (1955).

[9] V. E. Hart, J. Polymer Sci. 17, 215 (1955).

[10] J. H. Baxendale, S. Bywater, and M. G. Evans, J. Polymer Sci. 1, 237 (1946).

[11] B. Baysal and A. V. Tobolsky, J. Polymer Sci. 9, 171 (1952)

[12] E. W. Montroll and R. Simha, J. Chem. Phys. 8, 721 (1940).

Washington, October 10, 1955. 